XXIII.

Aus dem pathologischen Laboratorium zu Kiew

(Prof. W. Lindemann).

\title{
Experimentelle Untersuchung über den Einfluss der Resection des Plexus coeliacus auf die Veränderung der Langerhansschen Inselchen des Pankreas.
}

\author{
Von \\ Dr. P. Zagorowsky. \\ (Hierza Tafol XI.)
}

I.

Im Jahre 1907 veröffentlichte Pflüger im Band 118 seines Archivs einen Artikel unter dem Titel "Untersuchungen über den Pankreasdiabetes". In dieser Arbeit hält er sich eingehend über die Ursachen auf, welche den Pankreasdiabetes bedingen, wobei er bei seinem Entstehen die Bedeutung der inneren Secretion in Abrede stellt. Er schreibt: „Wenn ungeachtet aller negativ ausgefallenen Versuche die Lehre über die innere Secretion des Pankreas noch viele Anhänger aufzählt, so liegt der Grund hierfür in den von 0. Minkowski angestellten Versuchen über subcutane Pankreastransplantation." Pflüger erkennt jedoch die Beweiskraft dieser Versuche nicht an, und zum Schlusse des Kapitels „Kritische Prüfung der Hypothese der inneren Secretion des Pankreas" äussert er sich wie folgt: "Die bisherigen Forschungen haben also bewiesen, dass in den zahlreichen und sorgfältigen Arbeiten, welche der Begründung der inneren. Secretion gewidmet worden sind, auch keine Spur von Beweiskraft vorhanden ist." Indem nun Pflüger dem Pankreas eine innere Secretion abspricht, ist er zu gleicher Zeit ein Anhänger der Theorie der nervösen Abstammung des Diabetes. Er nimmt nämlich an, „dass sich die antidiabetische Kraft des Pankreas unter dem Einfluss des Nervensystems befindet" ${ }^{\prime}$. Aus dem Grunde aber, dass das Pankreas sowie die Leber aus der Endodermis, d. h. aus dem veränderten Teil der Wandung des Duodenums entsteht, glaubt er ferner in dieser Wandung des Verdauungsapparats ein centrales, nervöses Organ, reich an gangliösen Zellen, welches die Functionen der Drüse reguliert, zu ersehen. Zum Beweise dieses Satzes exstirpiert er bei einem gesunden Frosch denjenigen ganzen Abschnitt des Dünndarms, welcher mit dem Pankreas in Berührung steht, von der Pars pylorica des Magens ab, wobei die Drüse völlig intact bleibt. Als Resultat dieses Versuches beobachtet er einen Diabetes von solcher Stärke, wie er nie bei totaler Exstirpation des Pankreas zur Beobachtung gelangt. $\mathrm{Zu}$ demselben Resultat gelangt man nach der Resection des Mesenteriums zwischen Darm und Pankreas. Die Er- 
gebnisse dieser Versuche beweisen nach Pflüger unzweifelhaft, dass die Exstirpation des Duodenums die Entstehung des Diabetes genau ebenso beeinflusst wie auch die Exstirpation des Pankreas, und diese Tatsache findet eine Stütze darin, dass in den Wandungen des Duodenums ein selbständiges, antidiabetisches, nervöses, centrales Organ eingeschaltet ist. Pflüger schreibt: „Wie das Herz zu pochen fortdauert, wenn auch alle von aussen herantretenden Nerven zerstört werden, weil die darin gelegenen peripheren, nervösen, motorischen Centralorgane zu functionieren fortdauern, so auch das antidiabetische Centrum des Duodenums." Mit anderen Worten: Pflüger behauptet, dass die antidiabetische Kraft des Pankreas unter dem Einfluss der in der Wandung des Duodenums gelegenen Knoten sich befinde. Indem er nun diesen Gedankengang weiter entwickelt, gelangt er zu der Voraussetzung, dass die Exstirpation der Drüse eine Glykosurie vielleicht nur aus dem Grunde hervorruft, weil die antidiabetischen Nerven, welche vom Duodenum stammen und auf die Leber in dem Sinne einwirken, dass sie die Zuekerbildung verhindern, bei dieser Operation zerdrückt werden. Auf solche Weise bezweifelt er das Vorhandensein eines pankreatischen Diabetes. Er sagt: "So wie die von der Oblongata stammenden und zur Leber hin sich begebenden Nerven durch Anregung zur Entstehung einer Diastase die Zuckerbildung steigern, so verhindern die, vor allem vom Dünndarm ausgehenden, diabetischen Nervenfasern durch Anregung zur Bildung eines antidiabetischen Ferments die Zuckerbildung." Alle Versuche, welche daraufhingerichtet sind, das Vorhandensein eines duodenalen Diabetes zu beweisen, hat Pflüger an Fröschen angestellt. Er versuchte auch an Hunden zu experimentieren, fand aber, dass zur Durchführung dieser Versuche an Säugetieren eine grosse chirurgische Technik vonnöten sei. Seine Versuchstiere kamen nach zu kurzer Zeit nach der Operation um, so dass für ihn die Möglichkeit nicht vorlag, bei ihnen das Vorhandensein des Diabetes zu constatieren. Dieser Artikel hat die Frage nach dem duodenalen Diabetes angeregt; eine Frage, welcher Pflüger viel Zeit geschenkt hat. Schon im Bande 119 seines Archivs finden wir unter dem Titel: „Ueber die Natur der Kräfte, durch welche das Duodenum den Kohlenhydratstoffwechsel beeinflusst ${ }^{u}$, seinen der weiteren Ausarbeitung der Theorie des duodenalen Diabetes gewidmeten Artikel. In den betreffenden Versuchen bedient er sich Frösche, weil er letztere für diesen Zweck geeigneter hielt als Hunde, und zwar aus dem Grunde, weil bei Jetzteren das Duodenum dem Pankreas nicht dicht anliegt, und man daher mit dem Darm frei operieren kann. Seine Versuchsanordnung ist folgende: Um den Einfluss seitens der vom Duodenum zum Pankreas sich hinzichenden Nerven ohne Circulationsstörung in beiden Organen zu unterbrechen, unterbindet er das ganze Mesenterium fest mit seidener Ligatur zu einigen Bündeln; um eine Läsion der Gefässe zu vermeiden, legt er unter die Ligatur einen metallenen Draht, über welchem er darauf den Faden durchschneidet. Auf solche Weise ist es ihm gelungen, die Nervenleitung zu unterbrechen, ohne in den meisten Fällen eine Kreislaufsstörung zu verursachen. Auf Grund dieser Versuche gelangt er nun zu dem Schlusse, dass man nach Unterbrechung der Leitungs- 
fähigkeit derjenigen Nerven, welche das Pankreas mit dem Duodenum verbinden, Zuckerausscheidung beobachtet, welche bis zum Tode des Tieres währt. Letzterer tritt später ein, wenn das Tier im Juli operiert worden ist, und bedeutend früher, wenn die Operation zu Ende dieses Monats oder im August stattgefunden hat. Die Zuckerausscheidung ist in diesen Versuchen jedenfalls eine langdauernde, und darum nennt Pflüger die Folge der Resection des Duodenums duodenalen Diabetes. In zwei Versuchen blieb der Diabetes zwar ganz aus oder es waren bloss Spuren davon vorhanden. Pflüger findet aber eine Erklärung dieser Tatsache darin, dass diese Versuche Ende Juli oder Anfang August stattgefunden haben, d. h. zur Zeit, als im Körper des Frosches eine geringe Menge von Glykosen vorhanden war. Auf diese Weise entstand die Lehre vom duodenalen Dialıetes, dessen Grundsätze Pflüger in folgenden Worten zusammenfasst: „Die Exstirpation des Duodenums ruft beim Frosch einen Diabeles hervor, an Stärke denjenigen übertreffend, welcher nach der Entfernung des Pankreas entsteht. Dieser Diabetes tritt auch ohne Exstirpation des Duodenums oder des Pankreas ein, wenn man die Nerven und Blutgefässe durchschneidet, welche den Darm und die Drüse miteinander verbinden, oder, wenn man auf irgend ejnem anderen Wege das unmittelbare functionelle Verhältnis zwischen Darm und Drüsenaustausch zerstört und ferner, wenn ndie sogen. innere Drüsensecretion, welche eine antidiabetische Arbeit leistet, dem Einfluss derjenigen Nerven unterworfen wird, welche vom Duodenum her zum Pankreas hinleiten"; mit anderen Worten, Pflüger behauptet, dass im Duodenum ein selbständiges, antidiabetisches Centrum gelegen sei.

Die Frage nach dem Vorhandensein eines duodenalen Diabetes ist neu und nicht bewiesen; sie hat daher in der Jiteratur regen Meinungsaustausch seitens ihrer Anhänger (Zak, Herlitzka, Cavazzani, Sohlern u. a.), sowie vieler Gegner (Rosenberg, Ehrman, Eichler, Silbergleit, Visentini und besonders Minkowski) wachgerufen.

Minkowski resumiert seine Versuche folgendermassen: "Es unterliegt keinem Zweifel, dass die Exstirpation des Duodenums beim Hunde keinen Diabetes zur Folge hat; von einem nervösen, centralen Organ, welches in der Wandung des Duodenums gelegen wäre und dem Pankreas eine antidiabetische Kraft verleihe, kann keine Rede sein. " Was die innere Secretion des Pankreas anbelangt, so ist sie nach Minkowski einem regulierenden Einfluss seitens der Nervenbahnen unterworfen, und dieser Satz sei bis heute von keinem Anhänger der Lehre seiner specifischen Drüsensecretion widerlegt worden.

Ein jeder Forscher, der in diesem Gebiet tätig gewesen, hat seine Beweise und Ausführungen hauptsächlich auf Ergebnisse einer experimentalen Forschung gegründet. Wir wollen auf diese Frage nicht näher eingehen, weil das uns über den Rahmen unserer Aufgabe hinausführen würde. Wir weisen bloss darauf hin, dass wir aus unseren eigenen Ergebnissen den Schluss ziehen, dass die Glykosurie, welche bei der Resection des Duodenums beobachtet wird, von der Reizung des Plexus coeliacus, dem man bei der Operation ein Trauma beigebracht, abhängig ist. Dieser Satz wird auch von Pflügers Anhängern Zak und Herlitzka 
bestätigt, welche die Entstehung der Glykosurie auf Einfluss des sympathischen Nervensystems zurückführen. Was die Frage nach dem $\mathrm{Zu}$ sammenhang der Langerhansschen Inseln mit dem Diabetes betrifft, so ist sie ungeachtet einer grossen Anzahl ihr gewidmeter Arbeiten und der widerspruchswollen Resultate, $z \mathfrak{u}$ welchen die verschiedenen Forseher gelangt sind, als nicht endgültig gelöst zu betrachten. Da wir ausschliesslich die Langerhansschen Inseln und den sie regulierenden Einfluss des Plexus coeliacus im Auge haben, so werden wir auf die Literatur über den Bau des Pankreas überhaupt nicht eingehen, um so mehr, als sie zur Genüge von vielen Verfassern behandelt worden ist (Podwissozky, Jarotsky, Oser, Pischinger u. a.). Wir werden uns bloss auf die hervorragendsten Arbeiten über die Langerhansschen Inseln und ihre Innervation beschränken, da wir annehmen, dass eine solche Arbeit die Wirklichkeit der beiden, die Morphologie der Langerhansschen Inseln betreffenden Theorien noch besser begründen wird, nämlich: 1. die Theorie der anatomischen Präexistenz der Inseln und die Beständigkeit ihrer Zusammensetzung und 2. die Theorie ibrer functionellen Schwankungen. Die Anhänger der ersten Theorie sind: Kühne und Lea, Renaut, Sokolow, Ulezko, Dieckhoff, Sobolew, Vassale, Massari, Gianelli und Giacomini, Diamare, Jarotsky, Schultze, Opie, Stange, Schmidt u. a. Die Repräsentanten der zweiten Theorie: Saviotti, Lewaschew, Dogel, Harris und Gow, Pischinger, Kolosow, Laquesse, Mankowski, Dale, Gentes, Karakaschew u. a.

Langerhans, der die nach ihm benannten Bildungen im Pankreas beschreibt, äusserte sich, wie bekannt, in dem Sinne, dass dieses Nervenendungen wären, obgleich auch mit einem Widerruf, betreffs der Neigung damaliger Zeit, alles Unbekannte auf Nerven zu beziehen. Aber schon Kühne und Lea, welche an frischer und lebender Kaninchendrüse arbeiteten und unter dem Mikroskop die Abwesenheit eines Durchflusses, Reichtum an Gefässen, Windungen, der die Inseln bildenden Zellenstränge, beobachteten, sind zu der Schlussfolgerung gelangt, dass diese Inseln völlig selbständige, sui generis, Bildungen von lymphoidem Charakter sind. Ihren Ansichten schliesst sich auch Renaut an. In den Schleifen des reticulären Bindegewebes, behauptet er, seien von Gefässen umflochtene Zellen enthalten. Die Anhäufung solcher Zellen oder Inseln sind in Wirklichkeit kleine lymphatische Drüsen, welche im Pankreas eingeschlossen sind ( ${ }^{\text {un }}$ organ lympho-glandulaire ").

Diese Ansicht wurde schon im Jahre 1882 durch Podwysozky widerlegt, welcher, indem er nur eine äussere Aehnlichkeit der Inseln mit den Follikeln (daher sein Vorschlag, die Inseln Pseudofollikel zu nennen) anerkannte, sich consequent für die epitheliale Natur der Inselzellen aussprach und als erster in der Literatur dieser Frage die graphische Darstellung eines Inselchens gab, auf welchem die epitheliale Natur der sie bildenden Zellen deutlich ausgedrückt ist.

Später änderte Renaut selbst diese Ansicht über die Inselchen. Indem er auf die Abwesenheit einer besonderen Kapsel, auf die originelle Verteilung arterieller Gefässe in derselben, auf den epithelialen Charakter ihrer Zellen hinweist, kommt Renaut zu der Schlussfolgerung, dass die 
Inselchen vollständig fertige Bildungen sind, mit einem immer gleichen Bau und einer vermutlich inneren Function. Er gibt keine Erklärung ihrer physiologischen Bedeutung und weist nur darauf hin, dass nach ihrer Aehnlichkeit mit der Gl. pituitaria, thyreoidea u. a. und nach ihrer Entwicklungsgeschichte beurteilt, die Inselchen zweifellos eine sehr wichtige Rolle spielen.

In seinem ersten Artikel über das Pankreas bei den Amphibien nahm Ebner Abstand von einer Erörterung der Bedeutung der Langerhansschen Inselchen (er bemerkte u. a. die venösen Sinus um die Inselchen herum, was übrigens sich später nicht bestätigte), dann aber erktärte er sich für die Selbständigkeit ihres Bestandes und ihrer Function, welche den Kohlehydratwechsel regelte.

Um die Frage über die anatomische Selbständigkeit der Inseln $z u$ lösen, griff Gibbes zu den vergleichend anatomischen Forschungen des Pankreas bei verschiedenen Klassen von Tieren. Er fand jedoch in den Inselchen weder Kapseln noch Abführwege und kam zu dem Resultate, dass diese embryonale Ueberbleibsel sind, welche in einer gewissen Beziehung zu der Function des Pankreas stehen.

Bizzozero und Vassale bedienten sich zum Beweise der Selbstständigkeit der Inselchen der embryologischen Methode. In dem Fötus der Meerschweinchen fanden sie Inselchen und vermissten karyokinetische Figuren bei der Drüsenregeneration bei Erwachsenen.

Arnozan und Vailard kamen zu derselben Schlussfolgerung, als sie das Pankreas der Kaninchen und Massari das der Fische studierten. Als eine typische Drüse mit innerer Secretion spricht auch Brunn die Inselchen an, nachdem er die bereits vorhandenen Literaturergebnisse einer kritischen Prüfung unterworfen hatte. Gianelli und Giacomini beschäftigten sich mit der Untersuchung der Pankreasstructur bei allen Amphibien. Bezüglich der Inselchen weisen sie darauf hin, dass es Gebilde seien von deutlicher, röhrenförmiger Structur mit schmalem Lumen und von epithelialem Charakter; ibre Zellen enthalten Granula, wobei Kapseln und Abführungswege fehlen. Die genannten Verfasser betrachten sie als besondere, etwas in den Darm secernierende Partikelchen.

Helly untersuchte das Pankreas der Meerschweinchen und Selachier und schliesst aus seinen Forschungen, dass die Langerhansschen Inselchen Gebilde sui generis und dass sie aus dem Entoderm stammen. Uebergangsformen gebe es nicht und es sei nicht richtig, dass man sie für ein Reservematerial zur Regeneration des Pankreas ansieht. Sie seien wirkliche Drüsen von beständigem histologischem Aufbau. Als ein eifriger Verteidiger der anatomischen Beständigkeit steht Diamare da. Er hat ähnlich wie Gibbes die Inselchenstructur bei verschiedenen Tierklassen in ihrem embryonalen und postembryonalen Zustande studiert. Er behauptet, dass die Inselchen bei allen von ihm untersuchten Tieren vorkämen; bei den einen gänzlich abgesondert, bei den anderen in der Drüsenkapsel selbst eingeschlossen. Ein besonderes Bindegewebe der Umhüllung findet letztgenannter Autor bei diesen Inselchen nicht. Ebenso nicht Uebergangsformen zwischen den Partikelchen und Inseichen, sowie Abflusswege oder Lumina. Der Grund, wo ihre Zellelemente hineinge- 
schaltet sind, und das Kapillarnetz sei dasselbe, wie in dem umgebenden Drüsengewebe. Daher sei ihre Verbindung mit den Partikelchen die innigste; sie sei aber keinesfalls, was den Aufbau und die Function betreffe, mit dem übrigen Gewebe identisch. Indem er die Ansichten seiner Vorgänger einer scharfen Kritik unterzieht, kommt or zu dem Schlusse, dass die Langerhansschen Inselchen Bildungen seien, welche ausschliesslich für eine innere Secretion, gleich wie Gl. thymus, thyreoidea, suprarenales u. a. sorgen; sie sollen ferner einen ständigen Bestand und Structur sowie eine scharf ausgeprägte, anatomische Individualität besitzen.

Für Selbständigkeit der Inselchen, Abwesenheit von Kammern, Uebergangsformen und Ausführungswege tritt auch Poschon ein, dem eine reichlich gesammelte Literatur über die Langerhansschen Inselchen zur Verfügung steht. Alle bisher aufgezählten Verteidiger der Theorie einer anatomischen Präexistenz und Beständigkeit der Langerhansschen Inselchen stützen sich in ihren Schlussfolgerungen auf histologische oder embryologische Ergebnisse. Ferner folgt eine Reihe von Arbeiten, denen Experimente an Tieren verschiedener Art, wie Hunger, Unterbindung des Abfuhrweges der Drüse, Injection von Glykose usw., zugrunde liegen. Man erhoffte durch ähnliche Experimente beim Studium der unter solchen Bedingungen eintretenden morphologischen Veränderungen der Drüsenzellen ihre physiologische Rolle in das rechte Licht zu stellen. Von dieser Forschergruppe wäre vor allem Jarotzky zu erwähnen, der an weissen Mäusen experimentierte und gefunden hat, dass unter jeder Versuchsbedingung die Anzahl der Inselchen und die Grösse ibrer Zellen ungefähr die gleiche sei. Sie sondern sich scharf von dem übrigen Drüsengewebe $a b$, sie seien in Streifen, zwischen welchen Spalten zu beobachten seien, angeordnet. Einen Abfuhrweg habe Verfasser nie beobachtet. Ebenso leugnet er ihre partikelartige Structur. Die Inselchen haben nach Verfasser zwei Besonderheiten, welche sio von den übrigen Partikelchen unterscheiden: "1. ein deutliches Bindegewebe der Umhüllung und 2. Abwesenheit von Abführungswegen. Indem er nun ferner darauf hinweist, dass er keine Uebergangsstadien beobachtet habe, dass die Inselchen in einer unveründerlichen Anzahl bei verschiedener Art von Ernährung sich befinden, stimmt er Lewaschow und Statkewitsch nicht bei, sondern hebt hervor, dass die Langerhansschen Inselchen absonderliche selbständige in das Gewebe des Pankreas eingeschaltete Organe seien."

Betreffs ihrer physiologischen Bedeutung spricht sich Jarotsky in dem Sinne aus, dass die Inselchen an der Bildung einiger Fermente durch die Zellen teilnehmen, indem sie Substanzen ausscheiden, welche eines der chemischen Stadien in der Entwicklung des Ferments oder der Substanz darstellen, welche für die Zellen selbst nötig sind, damit in letzteren ein Ferment sich bilden könne. Man injicierte dem Tiere Pilokarpin oder Atropin oder man leitete in dic Vene Glykose hinein und versuchte auf diesem Wege der Lösung der Frage näher zu kommen.

Solchen Versuchen begegnen wir bei Jarotsky, Diamare, Sobolew, von welchen die Rede weiter unten sein wird, Lepine u. a. Endlich hat man seit der Erfindung der Phloridzin- und Adrenalinglykos- 
urie Versuche an Tieren durch Vergiftung mit den erwähnten Mitteln angestellt mit darauffolgender Untersuchung ihres Pankreas hauptsächlich zur Klarlegung der Langerhansschen Inselchen (Herter, Shedini, Vigliani, Lazarus, Heiberg, Tiberti u. a.). Die Untersuchungsresultate dieser Autoren sind widerspruchsvoll und wir werden darauf weiter unten im Zusammenhang mit unseren Ergebnissen zurückkommen.

Schultze und nach ihm Sobolew stellten fast gleichzeitig und unabhängig voneinander Versuche mit Unterbindung der Drüse oder ihrer Abfuhrwege an, wobei sie von der Voraussetzung ausgingen, dass, wenn tatsächlich eine äussere Function den Partikelchen eigen wäre und eine innere den Inselchen, so müssten bei der Unterbindung der Drüse nur diejenigen Elemente atrophieren, welche nicht functionieren. Da ist es nun Schultze an Schweinchen, Sobolew an Kaninchen, Hunden und Katzen gelungen, zu zeigen, dass bei der Unterbindung eines Teils der Drüse oder ihres Wirsungianschen Durchflusses nur die Partikelchen atrophieren, während die Inselchen unverändert bleiben. Auf Grund seiner Versuche und mikroskopischen Forschung der Drüse hält Schultze die Inselchen für Blutdrüsen, welche den Kohlehydrataustausch regulieren.

Sobolew sagt in der Schlussfolgerung seiner Dissertation: „Die Langerhansenschen Inselchen sind Elemente, welche sich anatomisch sowie functionell vom Verdauungsapparat unterscheiden .... Es sind Drüsen ohne Abführwege, und ihre Function besteht im Regulieren des Kohlehydratstoffwechsels im Organismus".

Den Durchflussweg bei Kaninchen unterband auch Sauerbeck. Interessant ist in seinen Versuchen die Tatsache, dass bei seinen Kaninchen nach der Unterbindung des Duct. Wirsungianus Glykosurie eintrat, was die übrigen Autoren nicht beobachtet und auch nachher nicht bestätigt haben. Sauerbeck gelangt zu dem Schluss, dass die Veränderungen der Inselchen und die Glykosurie einander parallel gehen.

Als eine der letzten Arbeiten in dieser Richtung ist die Untersuchung von Visentini zu beachten. Er unterband den Abfubrweg bei Kaninchen und Hunden. Bei Hunden, welche zu verschiedener Zeit nach der Unterbindung getötet waren, beschrieb er eine scharf ausgeprägte Sklerose und ein völliges Verschwinden der Drüsenpartikel bei voller Erhaltung und normalem Aussehen der Langerhansschen Inselchen. Einen solchen Zustand der Drüse hat er jedoch bloss bei zwei (von 24) Hunden constatiert, welche am 160. und 212. Tage getötet wurden. In den übrigen Zeiträumen sind, wie der Verfasser selbst eingesteht, die Partikelchen entweder normal oder wenig verändert. Bei dem am 212. Tage getöteten Hunde war eine leichte Glykosurie zu beobachten, bei den übrigen gar keine. Auf Grund dieser Versuche schliesst Visentini auf eine specifische Natur und Function der Inselchen. Ausser den Experimenten mit Unterbindung der Abfuhrwege sind von erwähnten Verfassern noch Versuche mit Kohlehydratfütterung, Glykoseinjection usw. angestellt. Sie fanden hierbei meistens Veränderungen, welche sie zugunsten ihrer Theorie deuteten.

So fand z. B. Sobolew eine Grössenabnahme der Inselchenzellen, Abnahme an fuchsinophilen Körnern usw. bei Ernährung der Tiere mit Zucker oder seiner Injection in die Vene und erklärte es dadurch, dass 
die Zellen unter solchen Umständen diejenigen Substanzen verbrauchten, welche die Zuckerverbrennung in den Geweben fördern.

Jarotsky beobachtete bei Kohlehydratfütterung Hyperplasie der Zellen, Zunahme der darin enthaltenen Körner und erklärt es durch gesteigerte, secretorische Tätigkeit.

Hierher gehören noch Diamare, Lazarus u. a.

Die dritte Verfassergruppe sucht Beweise für die Richtigkeit ihrer Theorie in pathologisch-anatomischen Ergebnissen. Die Begründer dieser Art Forschungen sind Opie in Amerika und Sobolew in Russland.

Opie beobachtete eine ausgeprägte hyaline Entartung der Inselchen in fünf Fällen von typischem Diabetes.

Max Herzog beschrieb fünf Fälle von typischem Diabetes, wo er kein einziges Inselchen vorfand, an ihrer Stelle aber ein narbiges Gewebe sich herausgebildet hatte. Sobolew veröffentlichte 17 Fälle von Pancreatitis bei Nichtdiabetikern und 15 bei Diabetikern. Bei letzteren ist entweder die Anzahl der Inselchen oder ihre Grösse vermindert, oder sie sind gänzlich verschwunden. Hierher gehören auch die Beobachtungen der Anhänger der Theorie der anatomischen Selbständigkeit der Inselchen: Wright u. Löslin, Gentes, Weichselbaum, Stangl, Curtis, Thoinot, Delamare u. a. Bei ihnen allen wiederholen sich die Hinweise darauf, dass bei dem Diabetes hauptsächlich die Inselchen betroffen werden. Das Betroffensein äussert sich entweder in der Verringerung ihrer Anzahl, oder in der Grössenabnahme ihrer Zellen, oder in ihrer Hyalinisation, Sklerose, in den darin vorgefundenen Blutergüssen usw.

Bis jetzt haben wir in Kürze die Ansichten der Vertreter der Theorie der histologischen Structur der Inselchen angeführt; jetzt halten wir einen kurzen Ueberblick über die Arbeiten der Anhänger der zweiten Theorie, ihrer functionellen Schwankungen. Der erste, welcher ihre anatomische Selbständigkeit anfocht, war Saviotti. Er behauptete unmittelbar nach Langerhans, indem er am Pankreas von Fröschen und Hunden arbeitete, dass sie Epitheliengänge zweiter Ordnung seien.

Als Hauptgegner der ersten Theorie ist Lewaschow anzusehen; seine Untersuchungsmethode ist die Heidenhains: Mikroskopische Analyse der Drüse in verschiedenen Verdauungsperioden. Die Hauptbasis, worauf er und seine Anhänger ihre Meinung begründen wollen, beruht auf Auffindung von Uebergangsformen zwischen den Partikel- und Inselchenzellen. Nach ihm sind die Langerhansschen Inselchen gewöhnliche Drüsenpartikelchen, welche einer gesteigerten Tätigkeit unterworfen sind. Dafür spricht ausser dem Vorhandensein der Uebergangsformen noch die Verbindung der Inselchen mit den Abfuhrwegen, sowie die Vermehrung ihrer Zahl nach Pilocarpinisation oder intensiver Ernährung und ihre Verminderung bei Ruhezustand der Drüse.

Bei einmaliger Ernährung sowie bei Hungerzustand sind ihrer wenig, sie vermehren sich aber bei wiederholter Nahrungsverabreichung. In der Ruheperiode nach der Verdauungstätigkeit bilden sich aus den Inselchen pankreatische Acini zurück.

Dogel untersuchte die Drüse bei Menschen, und da er in den Inselchen keine Abfuhrwege vorfand, nimmt er die Möglichkeit einer 
fettigen Degeneration an. Er kommt zu dem Schlusse, dass es Drüsenteile seien, welche eine regressive Metamorphose durchgemacht haben. In physiologischer Hinsicht seien es also rtote Punkte ${ }^{u}$.

Harris und Gow untersuchten die Langerhansschen Inselchen bei allen Klassen von Wirbeltieren (ausser Fischen) und ziehen den Schluss, dass es drei Inselchentypen gebe: 1. mit runden oder länglichen Zellen und mit einer Kapsel mit nicht bemerkbaren Grenzen zwischen den Zellen selbst, 2. mit nicht grossen durchsichtigen Zellen, deren Grenzen deutlich ausgeprägt sind, und 3. summierte Gruppen. Diese Bildungen seien secundärer Abkunft ( secondary cell groups"), und sie nehmen, aller Wahrscheinlichkeit nach, Anteil an der äusseren Secretion der Drüse.

Statkiewitsch untersuchte die Inselchen bei Hungertieren. Die Inselchen sind nach ihm ein Product der Veränderung der durch Hunger verkleinerten Partikelchen selbst (und nicht durch Tätigkeit, wie Le w a s ch ow meint). Die zahlreichsten Arbeiten gehören Laquesse an. Den Hauptwert dieser Arbeiten bildet die Fülle an embryologischen Ergebnissen. Der Entstehung nach unterscheidet Laquesse primäre und secundäre Inselchen. Letztere sollen aus pankreatischen Röhrchen entstehen und die Fähigkeit besitzen, in dieselben wieder zurückzukehren. Er beobachtete an ihnen Uebergangsformen, eine geringere Färbungsfähigkeit und kleinere Kerne; ihre Function soll die der inneren Secretion sein. Ihrer Natur nach seien sie nichts anderes als Drüsenteile, welche für eine gewisse Zeit zu Zwecken einer inneren Secretion sich verändert hätten. Eng schliesst sich Lewaschow Pischinger an, der in den Inselchen centroacinöse Zellen beobachtet zu haben glaubt. Die Inselchen seien Anhäufungen von Partikelchen, die nach lang dauerndem Functionieren einer Erholung bedürfen.

Pugnat schreibt den Drüsenzellen lymphoiden Charakter zu. Er beobachtete Uebergangsformen und erklärt sich für ihre Abstammung von gewöhnlichen secernierenden Partikeln; er geht so weit, dass er ihnen die Fähigkeit zuschreibt, Zymogen in Trypsin (durch die Milz) zu.verwandeln. In der russischen Literatur sind zwei Dissertationen auf die Theorie der functionellen Schwankungen der Inselchen aufgebaut; diese sind: die Minkowskis und Tschassownikows.

Der erstere von ihnen vermutet, hauptsächlich auf Grund der Experimente und der vergleichend histologischen Forschungen, dass die Langerhansschen Inselchen "zeitweilig veränderte Partikelchen des Pankreas" seien. Sie stehen im Zusammenhang mit den Abfuhrwegen, haben ein gemeinsames Capillarnetz, keine Kapseln und stellen verschiedene Uebergangsformen mit Partikelzellen dar. Ihre Anzahl ist variabel, abhängig von der Ruhe und Tätigkeit der Drüse, und in physiologischer Hinsicht stellen sie eine der Stadien der Drüsentätigkeit dar (Stadium der Langerhansschen Inselchen).

Tschassownikow meint, indem er sich auf die bistologischen Ergebnisse stützt, dass die Inselchen nicht selbständige Bildungen scien, wohl von Partikeln stammen, aber nicht mehr in dieselbe zurückkehren. Kapseln und Abfuhrwege seien nicht vorhanden. In functioneller Hinsicht seien es Drüsen mit innerer Secretion. Ausser den genannten Autoren 
gehören hierher: Dale, der die Uebergangsformen bei Hunden, Katzen und Kaninchen beschrieben, Gentes, Perdigreat und Tribondeau bei weissen Mäusen, Karakaschew u. a. Die Anhänger der Theorie ${ }_{n}$ der functionellen Schwankung " (balancement) wandten sich zu ihrer Begründung auch zu den pathologisch-anatomischen Forschungen. Sie beobachteten einige tiefe Verletzungen des Pankreas ohne Glykosurie: Hansemann, Contani u. a. Restmann untersuchte die Inselchen und kam zu dem Schlusse, dass der inneren Secretion die ganze Drüse untergeordnet sei, d. h. die Acini wie auch die Insulae. Hansemann, Gutmann, Karakaschew, Herxheimer, Schmidt u. a. meinen, dass die Hauptrolle im Diabetes die Acini spielten. Dasselbe muss man auch betreffs der experimentellen Prüfung der Theorie sagen. Hansemann z. B. beobachtete, nachdem er die Drüse in toto unterbunden hatte, in derselben Veränderungen wie in den Acini, so auch in den Inselchen.

Minkowski unterband den Durchflussweg bei Meersehweinchen: Veränderungen in den Acini und Inselchen. Laquesse sah Verletzung der Inselchen, wenn auch weniger bei der Unterbindung des Durchflussweges. Lombrosos Arbeiten mit Unterbindung dagegen zeigten, dass sogar nach 140 Tagen die Acini sich gut erhalten hatten. Gewöhnlich trat Sklerose ein, jedoch keine starke, und wenn sich auch Veränderungen zeigten, ebenso viel in den Acini wie in den Inselchen. Der Autor unterband den Durchflussweg bei 41 Hunden und untersuchte die Drüse zu verschiedener Zeit. Auch Pensa fand bei Unterbindung bei Kaninchen schwere Verletzungen in den Inselchen.

Was die Innervation der Langerhansschen Inselchen betrifft, so wissen wir von denselben folgendes: Im Jahre 1899 notierte Laquesse in seiner Arbeit, dass bei einem erwachsenen Schaf der periacinöse Plexus in einigen Punkten die Langerhansschen Inselchen erreicht hatte, jedoch auf ihrer Oberfläche stehen geblieben war, obne in dieselben hineinzudringen. Auch Diamare spricht von einem Hineindringen der Nervenbündel in die Langerhansschen Inselchen. Im Jahre 1902 beschrieb Gentes zum erstenmal bei der weissen Ratte das Vorhandensein eines periinsulären Netzes, d. h. eines Inselnetzes, von wo aus Fasern gehen, die, nachdem sie in die Substanz der Inselchen hineingedrungen sind, daselbst ein Netz bilden und endlich zwischen den Inselchenzellen mit den freien Enden, einem Knöpfchen, auslaufen. Das Intrainselgewebe scheint reicher zu sein als das Periinselgewebe. Jedoch Nervenzellen in diesem Gewebe zu erblicken, gelang Gentes nicht.

Im Jahre 1904 erschien die Arbeit Pensas, welche bewies, dass bei allen Wirbeltieren die I,angerhansschen Inseln eine Fülle von Nerven besitzen. Aus dem zusammengepressten periinsularen Plexus gehen feine, sehr gewundene Fasern aus, welche eine Menge Nebenzweige von sich geben; die letzteren bilden ein dichtes Netz um die Insel herum. Eine recht grosse Anzahl Nerven begleitet auch die Capillaren zwischen den Inseln. Ausserdem dringen auch die Nerven in das Innere der Inseln, wo sie sich teilen und zwischen den Zellen verflechten, so dass sie ein sehr dichtes Netz bilden. 
Auch bei den Amphibien, Reptilien und Vögeln constatierte Pensa das Vorhandensein eben solcher dichter Nervennetze. Bei den Schlangen endigen die zahlreichen Verzweigungen auf der Peripherie der Inseln büschelartig, wodurch sie dem periinsularen Plexus einen eigentümlichen Charakter verleihen. Bei den Säugetieren erhalten die Langerhansschen Inseln Nervenfasern aus dem perivasculären und periacinösen Plexus. Letzterer ist in den Inseln reicher als zwischen den Acini; er begleitet die Inseln. Indem wir uns mit der oben erwähnten Literatur begnügen und das oben Klargelegte resümieren, kommen wir zu Folgendem: 1. die Frage über die anatomische Individualität der Langerhansschen Inseln bleibt offen; 2. bei allen Wirbeltieren besitzen die Langerhansschen Inseln eine Fülle von Nerven, welche sich auf folgende Art verteilen: Auf dem Niveau der Inseln vermischt sich der perivaseuläre innig mit dem periacinösen Plexus, um anfangs den periinsularen Plexus und dann den intrainsularen zu bilden; letzterer begleitet die Gefässe, umspinnt sie ebenso wie auch die Inseln selbst und dringt zwischen die Inselzellen. Der periacinöse Plexus, sowie der perivasculäre werden jedoch aus Fasern gebildet, welche vom Plexus coeliacus ausgehen, der mit den Nebenplexus des Bauches verbunden ist, welche folgende Arterien begleiten: Art. splanchnica, mesenterica superior und hepatica. In den Stämmen des Plexus coeliacus, wie er heutzutage anerkannt wird, existieren Fasern aller Art: sensible, motorische, vasomotorische und sekretorische, folglich muss die Tätigkeit der Langerhansschen Inseln sich unter dem Einfluss des Plexus coeliacus befinden. Dieser Satz wird auch durch die pathologisch-anatomischen Ergebnisse begründet, die in meiner Arbeit „Zur Frage von den gegenseitigen Beziehungen zwischen Nervensystem und Zuckerkrankheit ${ }^{4}$ angeführt sind, und auf die ich bier die sich Interessierenden hinweise. Experimentelle Forschungen jedoch gibt es in dieser Richtung nicht; und das ist der Grund, weshalb ich es wagte, noch einmal das Pankreas der Tiere einer experimentellen Forschung zu unterwerfen im Sinne eines Studiums der Langerhansschen Inseln und zu versuchen, mich der Lösung der Frage über die Einwirkung der Resection des Plexus coeliacus auf die Veränderung der Langerhansschen Inseln zu nähern.

II.

Die Untersuchungsobjekte waren Meerschweinchen und Kaninchen als Vertreter der Grasfresser, weisse Ratte und Hund als Vertreter von Tieren, welehe van gemischter Nahrung leben. Es wurden zur Untersuchung nur gesunde und muntere Tiere mittleren Alters ausgesucht. Ausgeführt wurden die Versuche hauptsächlich in Morphiumnarkose, welche 1 Stunde vor der Operation in 1 proc. Lösung nach Berechnung $(1 \mathrm{ccm}$ auf 1 Kilo des Gewiehts) injieiert wurde. Während der Operation aber griff man zur leichten Chloroformnarkose, welche in dem Falle verstärkt wurde, wenn der Hund unruhig war. Bei der Laparotomie wurde der Schnitt am Rande der linken geraden Muskeln vorgenommen, die Därme wurden aus der Bauchhöhle herausgenommen, der Magen nach oben gezogen. Nachdem man die linke Niere, welche als Erkennungspunkt diente, ge- 
funden hatte, fand man auch leicht die Arteria coeliaca und die Arteria mesenterica superior und über ihnen wurde durch das Bauchfell der Plexus coeliacus sichtbar, der dann reseciert wurde. Die Section erfolgte gleich nach dem Tode des Tieres.

Kleine Stücke der Drüse, welche aus dem eben getöteten Tier genommen wurden, wurden teils nach den von Holmgren (Dreichloressigdreichlormilchsäure, Mischung Carnay u. a.), teils nach denselben nur etwas modificierten Methoden (z. B. zu der Dreichloressigsäure $6 \mathrm{pCt}$. wurde ein gleiches Quantum Chrom- $[1 / 4-1 \mathrm{pCt}$. $]$ oder Sublimatlösung gegeben) durchgeführt. Es wurden auch andere Bearbeitungsmittel angewandt, wie Fols und Flemmings Flüssigkeit u. a., jedoch als die bequemsten erwiesen sich die Mischungen der Dreichloressigsäure mit Chrom und Sublimat wie auch die Mischung von Sublimat, Alkohol und Essigsäure.

Die Objecte, welche man mit einer von den genannten Flüssigkeiten im Laufe von 18--24 Stunden bearbeitete, wurden im Wasser gewaschen und in Spiritus gelegt, dessen Stärke vermehrt wurde. Nach genügender Verdichtung wurden die Drüsenstücke mit Paraffin nach allgemeinbekannten Regeln begossen und in Schnitte zerlegt. Bei dieser Begiessungsart war die weitere Bearbeitung der Schnitte jedoch recht mühevoll, denn bei Befreiung der Schnitte vom Paraffin zerfielen dieselben nicht selten in kleine Stücke, mit denen es schwer zu manipulieren war; man musste die Stücke auf Glas kleben und dann in diesem Zustand sie einer weiteren Bearbeitung unterwerfen; aber auch diese Art hat ihre Unbequemlichkeiten. Um die Behandlung der Schnitte zu erleichtern, verfuhr ich wie folgt: Nach der Verdichtung der Pankreasstücke in Spiritus wurden sie in ganz leichte Celloidinlösung gelegt, von dort in Xylolparaffin und endlich in reines Paraffin. Auf diese Weise fielen sogar sehr dünne Stücke nicht auseinander und es war leieht, sie zu behandeln. Nachdem sie von Paraffin befreit waren, wurden sie gefärbt. Das Färben der Schnitte, welche man aus der Drüse gewonnen hatte, die mit Dreichloressig- oder Dreichlormilchsäure bearbeitet worden waren, geschah durch Weigerts Fuchsin; bei der Bearbeitung mit Flüssigkeiten, welche Osmiumsäure enthielten, wurden die Schnitte mit Safranin und PikroIndigo-Carmin gefärbt, bei der Bearbeitung aber mit Sublimat und Säurenmischung mit Chrom wurde doppeltes Färben mit Tionin und Fuchsin vorgenommen. Nach der Färbung wurden die Stäcke in reinen Spiritus oder mit einer Beimengung von Pikrinsäure gelegt und von dort auf das Objectglas. Dadurch, dass der Schnitt mit Hilfe von Löschpapier an das Glas gedrückt wurde, wurde er trocken, und es wurde darauf ein Tropfen Nelkenöl aufgegossen, welches ihn von dem Celloidin befreite; das Oel wurde wieder entfernt durch Aufdrücken von Löschpapier, und das Präparat wurde dann in Xylolbalsam gelegt.

III.

Zur mikroskopischen Untersuchung wurden aus dem Pankreas kleine Stücke Drüse genommen. Der Untersuchung der genommenen Stücke wollen wir zur Vergleichung mikroskopischer Bilder eine kurze Klarlegung des 
Inselchenbaues im normalen Pankreas der obengenannten Tiere vorausschicken, wie sie bei unseren Präparaten sich zeigte. Man muss noch hinzufügen, dass, um die Möglichkeit zu gewinnen, eine richtige Schätzung der beobachteten, mikroskopischen Veränderungen zu machen, ebenso auch, um eine wahrscheinlichere Schlussfolgerung zu ziehen, wir uns bemühten, Pankreasstücke bei Tieren von denselben Gebieten zu entnehmen. Dabei bestrebten wir uns, die Stücke unter gleichen Bedingungen zu bearbeiten und dasselbe bei der Färbung der Stücke der Schnitte zu beachten.

Die Langerhansschen Inselchen traten auf in Form von rundlichen oder ovalen Bildungen. Die Anzahl der die Inselchensubstanz bildenden Zellen war nicht immer dieselbe: zuweilen waren es nicht mehr als 3 oder 4, dann aber traten Inselchen auf, welche eine Anhäufung von bedeutender Anzahl von Zellen darstellen. Die Umrisse der letzteren sind verschieden: es gibt solche von conusartiger, pyramidaler Form, dann wieder sind sie polygonal, rundlich, cylinderförmig oder endlich unregelmässig. Ebenso verschieden ist auch ihre Grösse, aber im allgemeinen treffen sich an der Peripherie der Inselchen grössere Zellen als in den centralen Teilen. Die Conturen der Zellen sind verhältnismässig selten scharf ausgeprägt, in den meisten Fällen sind sie nicht ganz deutlich. Die innere Structur der Inselchenzellen trat nicht gleichmässig deutlich in den Zellen verschiedener Inselchen hervor. So konnten wir oft in mehr oder weniger isoliert stehenden Zellen neben einer feinen schwachen Granulation, die sogar bei homogenen Objectiven bemerkbar ist, grössere Körner unterscheiden, welche jedoch nie an Grösse den zymogenen Körnern (Fig. 1 und 2) gleichkamen. Diese grösseren Körnchen sind in einigen Zellen nur in geringem Masse den kleinen beigemischt, in anderen dagegen bilden sie den Hauptbestand des Zellkörpers.

Der Kern der Inselchenzellen hatte eine kugelförmige Gestalt, war hell, blasenartig, und seine Chromatinsubstanz war auf seiner Peripherie in Form von einigen Knäulchen verteilt. Einen Abfuhrweg oder eine besondere Hülle konnte man bei den Langerhansschen Inselchen nicht constatieren.

Nach der Resection des Plexus coeliacus stellten sich uns die Langerhansschen Inselchen auf unseren Präparaten in folgendem Bilde dar (Fig. 3 und 4): Die Inselchen sind verkleinert; das Protoplasma ihrer Zellen ist sehr schwach gefärbt; es gibt Stellen, wo die Inselchenzellen fast durchsichtig sind. Die Granulation ist weniger bemerkbar als in der Norm; grössere Körnchen fehlen ganz. Die Kerne der Inselchen sind arm an Chromatin; es sind nur ungefähr $2-3$ Körner geblieben. Im allgemeinen liegen die Kerne dichter nebeneinander als in der Norm und sind bedeutend eingeschrumpft; sie haben ihre ursprüngliche runde Form verloren, sind eckig geworden oder haben unregelmässige Umrisse angenommen. Die Capillaren in den Inselchen sind erweitert und mit Blutkörperchen angefüllt. Die acinösen Zellen des Pankreas wiesen nach der Resection des Plexus coeliacus keine Abweichungen von der Norm auf. 
IV.

Indem wir die von uns gefundenen Veränderungen der Langerhansschen Inselchen nach der Resection des Plexus coeliacus resumieren, können wir folgendes constatieren:

1. Nach der Resection des Plexus coeliacus bemerkt man in den Zellen der Langerhansschen Inselchen deutliche und recht charakteristische mikroskopische Veränderungen:

2. Diese Veränderungen betreffen die innere Structur des Protoplasmas, sowie auch der Kerne der Inselchenzellen und sind auf eine Verminderung der Granulation des Protoplasmas und auf die Einschrumpfung der Kerne zurückzuführen.

Zum Schluss halte ich es für meine angenehmste Pflicht, dem hochverehrten Herrn Professor W. Lindemann meinen innigen Dank auszusprechen. Er ermöglichte mir die Ausführung dieser Arbeit in dem von ihm verwalteten Laboratorium der allgemeinen Pathologie an der Universität St. Wladimir. Dem Professor der Histologie Herrn K. Miram danke ich herzlich für die Ratschläge, Hinweise, wie auch für die seltene Herzlichkeit und Aufmerksamkeit, mit denen er immer unabänderlich meine Arbeit leitete.

\section{Erklärung der Abbildungen auf Tafel XI.}

Figur 1 and 2. Zwei Typen der Inselzellen aus dem Pankreas. Färbung mit Tionin und Fuchsin. Die Zellen sind gemischt violett gefärbt; man bemerkt darin zwei Arten von fuchsinophilen Körnern. Vergr. Seitz Ocular IV. Objectiv $7 \mathrm{~mm}, 1 / 16$.

Figur 3 und 4. Dieselben Zellen nach Resection des Plexus coeliacus.

\section{Literatur.}

1. Arnozan et Vailard, Archives de physiol. norm. JIl. sér. 1884. T. 3.

2. Brachet, Journ. de l'anat. et de la physiol. 1892. T. 32.

3. Brunn, Ergebnisse der Anatomie und Entwicklung. 1895. Bd. 4.

4. Cantani, H., Diabete. Napoli 18i5. Cit. nach Lombroso.

5. Cavazzani, Zur Physiologio des Duodenums. Centralbl. f. Physiol. 1908. Bd. 22. Nr. 12.

6. Curtis et Gellé, Compt. rend. soc. de biol. Paris 1905.

7. Diamare, Anatomischer Anzeiger. 1899. Bd. 16. - Derselbe, Internationale Monatsschr. f. Anat. und Phys. 1899. Bd. 19. - Derselbe, Ebendas. 1905. Bd. 22. H. 4-6.

8. Dieckh off, Inaug.-Diss. Leipzig 1895.

9. Dogel, Arch. f. Anat. u. Physiol. 1893. Bd. 117.

10. Ebner, Arch. f. mikr. Anat. 1872. Bd. 8.

11. Ehrmann, Ueber den Einfluss der Ausschaltung des Zơölffingerdarms auf Zuckerausscheidung und seine Beziehung zum experimentellen Pankreasdiabetes. Archiv. f. d. gesamte Physiol. 1907. Bd. 119.

12. Fichler und Silbergleit, Ueber Glykosurie, experimentell hervorgerufen durch Verätzung und Verschorfung der Innenfläche des Darmes. Berliner klin. Woohen. schrift. 1907. Nr. 25.

13. Ellenberger, Vergleichende Histologie der Haussäugetiere. Berlin 1887. 
14. Frougoni et Stradiotti, Arch. ital. de biol. LI. II.

15. Gentes, Morphologie et structure des ilots de Langerhans etc. Thèse de méd. Bordeaux.

16. Gianelli ot Giacomini, Comunic. scient. d. B. Accad. di Siena. 1896.

17. Gibbes, Journ. of microsc. science. Vol, 24. p. 183. Cit. nach Minkowsky.

18. Gutmann, Virch. Arch. 1903. Bd. 172 und 1904. Bd. 177.

19. Hansemann, Verh. d. deutsch. path. Gesellsch. 1901. Bd. 187.

20. Harris and Gow, Journ. of physiol. Vol. 115.

23. Heiberg, Münchener med. Wochenschr. 1907. - Derselbe, Centralbl.f.d.ges. Phys. etc. 1907. Nr. 8.

21. Helly, Arch. f. mikroskop. Anat. u. Entw. 1905. Bd. 68.

22. Herlitzka, Ein Beitrag zur Kenntnis des Plügerschen Duodenaldiabetes. Arch. f. d. ges. Phys. 1908. Bd. 123.

24. Herter und Wakemann, Virch. Arch. Bd. 169.

25. Herzog, Virch. Arch. 1902. Bd. 168.

26. Jarotsky, Ueber die Veränderung der Grösse und der Structur des Pankreas unter Einfuss des Hungers. Dissert. 1898.

27. Karakascheff, Deutsches Arch. f. klin. Med. 1904. Bd. 82.

28. Korinfsky, Arch. f. Veterin.-Wissenschaften. 1910. Bd. 6.

29. Krause, Die Anatomie des Kaninchens. Leipzig.

30. Kühne und Lea, Untersuchungen aus dem physiol. Institut der Universität Heidelberg. 1882. Bd. 2. H, 4.

31. Labbé, M. et P. Thaon, La Presse méd. 1910. Nr. 62.

32. Layuesse, lbidem. 1910. 'T. 49.

33. Lazarus, Münchener med. Wochenschr. 1907. Nr. 45.

34. Lemoine et Lannois, Arch. de méd. expér. 1891. T. 3.

35. Lepine, Comp. rend. soc. de biol. 1903/4. T. 55.

36. Lewaschew, Arch. f. mikrosk. Anat. 1886. Bd. 26.

37. Lombroso, Ergebn. der Physiol. 1910. Bd. 9.

38. Lunin, Aerzte-Zeitung. 1907.

39. Marassini, Rivista di fisica, mat. e scien. nat. Vol. 8. No. 96 und 1908. Vol. 9.

40. Massarin, Rendic. della R. accad. dei Lincei. 1898. Vol. 7.

41. Minkowski, Die Totalexstirpation des Duodenums. Arch. f. exper. Path. uud Pharm. 1908. Bd. 58. - Derselbe, Weitere Mitteilungen über den Diabetes mellitus nach Exstirpation des Pankreas. Berliner klin. Wochensehr. 1892. Nr. 26. - Derselbe, Untersuchungen über den Diabetes mellitus nach Exstirpation des Pankreas. Arch. f. exper. Path. und Parm. Bd. 31. - Derselbe, Diabetes mellitus und Pankreas. Berliner klin. Wochenschr. 1890. Nr. 8.

42. Mouret, Journ. de l'anat. et de la phys. T. 81.

43. Nischbizky, Medicinische Rundschau. 1910. Bd. 73.

44. Opie, Bull. of the John Hopk. Hospit. 1900. Vol. 11 und Journ. of exper. med. 1900/1901. Vol. 5.

45. Pflüger, Untersuchungen über den Pankreasdiabetes. Arch. f. d. ges. Physiol. 1907. Bd. 118. - Derselbe, Ueber die Natur der Kräfte, durch welche das Duodenum den Kublenhydratstofwechsel beeinfusst. Ebenda. 1907. Bd. 119. Derselbe, Bemerkungen zu R. Ehrmann, Exstirpationen des Duodenums. Ebenda. 1907. Bd. 119.

46. Ponsa, cit. nach Laquesse.

47. P is chinger, Inaug.-Diss. München.

48. Podwisozky, Neue Daten über die Structur des Pankreas. Kiew 1882.

49. Poschon, cit. nach Korynfsky.

50. Pugnat, Journ. de l'anat. et de la phys. T',33 u. Comp. rend. de la soc. de biol. ''. 48. 
Zeitschrift f. exper. Pathologie u. Therapie. 16. Bd.

Taf. XI.

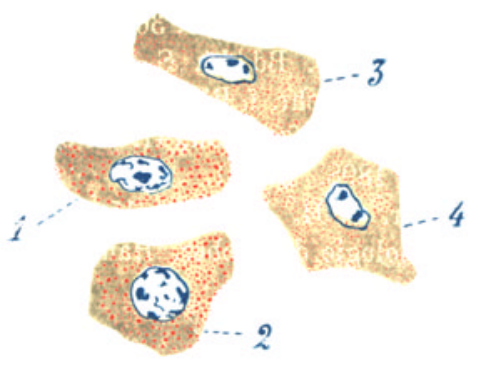

